\title{
Cascaded Parametric Amplification for Highly Efficient Terahertz Generation
}

\author{
Koustuban Ravi, ${ }^{1,3,4 *}$ Michael Hemmer, ${ }^{1}$ Giovanni Cirmi, ${ }^{1,2}$ Fabian \\ REICHERT, ${ }^{2}$ DAMIAN N. SCHIMPF, ${ }^{1,2}$ OliVER D. MÜCKE, ${ }^{1}$ AND Franz $X$. \\ KÄRTNER ${ }^{1,2,4}$ \\ ${ }^{1}$ Center for Free-Electron Laser Science, Deutsches Elektronen-Synchrotron (DESY), Hamburg, Germany \\ ${ }^{2}$ Department of Physics and the Hamburg Center for Ultrafast Imaging, University of Hamburg, Germany \\ ${ }^{3}$ Department of Electrical Engineering and Computer Science, Massachusetts Institute of Technology, Cambridge, MA, USA \\ ${ }^{4}$ Research Laboratory of Electronics, Massachusetts Institute of Technology, Cambridge, MA, USA \\ *Corresponding author: koust@mit.edu
}

Received XX Month XXXX; revised XX Month, XXXX; accepted XX Month XXXX; posted XX Month XXXX (Doc. ID XXXXX); published XX Month XXXX

A highly efficient, practical approach to high-energy multi-cycle terahertz (THz) generation based on spectrally cascaded optical parametric amplification (THz-COPA) is introduced. Feasible designs are presented that enable the $\mathrm{THz}$ wave, initially generated by difference frequency generation between a narrowband optical pump and optical seed $(0.1-10 \%$ of pump energy), to self-start a cascaded (or repeated) energy down-conversion of pump photons in a single pass through a single crystal. In cryogenically cooled, periodically poled lithium niobate, unprecedented energy conversion efficiencies $>8 \%$ achievable with existing pump laser technology are predicted using realistic simulations. The calculations account for cascading effects, absorption, dispersion and laserinduced damage. Due to the simultaneous, coupled nonlinear evolution of multiple phase-matched three wave mixing processes, THz-COPA exhibits physics distinctly different from conventional three-wave mixing parametric amplifiers. This in turn governs optimal phase-matching conditions, evolution of optical spectra and limitations of the nonlinear process. Circumventing these limitations is shown to yield conversion efficiencies $>10 \%$.

\section{(C) 2016 Optical Society of America}

OCIS codes: (320.7110) Ultrafast nonlinear optics; (260.3090) Infrared, Far; (190.0190) Nonlinear optics; (190.4970) Parametric oscillators and amplifiers

$\mathrm{DOI}$

Multi-cycle or narrowband terahertz (THz) frequency sources with simultaneously high pulse energies $(>10 \mathrm{~mJ})$ and peak fields
$(>100 \mathrm{MV} / \mathrm{m})$ in the frequency range between 0.1 and $1 \mathrm{THz}$ are of great interest for compact charged particle acceleration [1-2], coherent X-ray generation [3], linear and nonlinear spectroscopy and radar applications. Such a class of $\mathrm{THz}$ sources has the potential to transform current charged particle accelerator technology and enable unprecedented modalities in biomedical imaging, therapy and protein structure determination [3]. Among existing THz generation methods, photoconductive switches [4], are challenging to scale to high pulse energies. Vacuum electronic devices [5] are limited to low frequencies and peak powers while free-electron lasers are relatively expensive [6].

Due to the increase in laser pulse energies produced by solidstate laser sources, laser-driven narrowband $\mathrm{THz}$ generation methods based on difference-frequency generation (DFG) are attractive. However, an issue that needs to be addressed is the realization of high optical-to-THz energy conversion efficiency (or conversion efficiency), particularly at high pump energy. To generate tens of millijoules (mJs) of THz energy from Joule-class lasers, conversion efficiencies $>1 \%$ are necessary. On the contrary, conversion efficiencies of current multi-cycle sources have not exceeded the sub-percent level. Furthermore, no feasible proposals to achieve such high conversion efficiencies have been reported.

Previous work on multi-cycle THz generation demonstrated conversion efficiencies in gallium arsenide (GaAs) of $10^{-4}$ [7-8], in gallium phosphide $10^{-6}$ [9], and organic materials $10^{-5}$ [10]. In lithium niobate (LN), multi-cycle $\mathrm{THz}$ generation by interfering chirped and delayed copies of a pulse with tilted-pulse-fronts (TPF) was demonstrated [11]. However, TPFs have angular dispersion induced limitations [12]. Such issues may be circumvented by collinear $\mathrm{THz}$ generation in periodically poled lithium niobate (PPLN) crystals. However, the highest conversion efficiency achievable with such structures is only 0.1\% [13] due to large walk-off between the pump pulse and generated $\mathrm{THz}$ 
radiation. In order to obtain significantly larger conversion efficiencies, a feasible method which circumvents walk-off, minimizes loss and preserves phase-matching to enable a large number of repeated energy down-conversions of pump photons to THz photons (or cascading [14]) is required.

Here, we introduce a practical approach which involves using a strong narrowband (hundreds of ps transform-limited (TL) duration) optical pump pulse along with a narrowband seed, shifted in frequency from the pump by the desired THz frequency. The seed contains significantly lower pulse energy $(\sim 0.1-10 \%$ of pump). Long pump pulses alleviate walk-off between optical and $\mathrm{THz}$ radiation, nonlinear phase accumulation and permit higher energy pumping while limiting laser-induced damage. The initial DFG between these pulses generates narrowband $\mathrm{THz}$ radiation which then simultaneously drives the repeated generation of optical lines, each successively red-shifted by the THz frequency, to self-start a dramatic cascading process. Exponential THz energy growth over a single pass through a single crystal thus results. Since the rate of exponential growth is parameter dependent i.e. on pump intensity, THz frequency etc., the approach is termed $\mathrm{THz}$ cascaded optical parametric amplification (THz-COPA).

A conversion efficiency $>8 \%$ from collinear $\mathrm{THz}$ generation in a single pass through a single cryogenically cooled PPLN crystal is predicted, which is unprecedented even theoretically. For a pump pulse energy of $1 \mathrm{~J}$, the corresponding seed energy would span the $\mathrm{mJ}-100 \mathrm{~mJ}$ range. Such sources are readily available at $\mathrm{kHz}$ repetition rates with $1 \mu \mathrm{m}$ laser technology [15]. Furthermore, the required crystal apertures for Joule-level pulses with durations of hundreds of ps are $<1 \mathrm{~cm}^{2}$, which is attainable with existing technology [16].

THz seeded parametric amplification [17] was demonstrated in a non-collinear geometry in LN at room temperature. Optically seeded approaches in oscillator configurations [18-19] were demonstrated at room temperature. Cavity-based configurations limit the number of cascades that the pump may undergo while $\mathrm{THz}$ seeded approaches suffer from limited power available at $\mathrm{THz}$ frequencies.

A sequential combination of optical parametric amplifiers, has been previously been referred to as COPA [20] (with no relation to $\mathrm{THz}$ generation). However, that work is still a conventional threewave mixing process. On the contrary, the process described involves the simultaneous, coupled nonlinear evolution of multiple phase-matched three wave mixing processes (or multiple triplets of waves). We thus discuss the distinctive evolution of optical spectra in THz-COPA and deduce preferred phase-matching conditions and resulting limitations.

For the case under consideration, the optical spectrum consists of narrowband frequency distributions centered on various frequencies $f_{m}=f_{0}+m f_{T H z}$, where $f_{0}$ is the optical pump frequency, $m$ is an integer and $f_{T H z}$ is the generated $\mathrm{THz}$ frequency. Since the frequency distributions are narrowband, we may approximate them as discrete lines (or modes) in frequency space. The discrete modes are characterized by amplitudes $E_{m}(z)=A_{m}(z) e^{-j k_{m} z}$ where $A_{m}$ is the envelope, $k_{m}$ is the wave number of mode $m$. The evolution of $A_{m}(z)$ along the crystal length $z$ is given by

$$
\begin{aligned}
& \frac{d A_{m}}{d z}=\frac{-j 2 f_{m} \chi_{0}^{(2)}}{c n\left(f_{m}\right)}\left[A_{m+1} A_{T H z} e^{-j\left(k_{m+1}-k_{T H z}-k_{m}+2 \pi \Lambda^{-1}\right) z}\right. \\
& \left.+A_{m-1} A_{T H z} e^{-j\left(k_{m-1}+k_{T H z}-k_{m}+2 \pi \Lambda^{-1}\right) z}\right]
\end{aligned}
$$

The first term on the right-hand side (RHS) of Eq. (1) corresponds to DFG between the $(\mathrm{m}+1)^{\text {th }}$ optical mode and the $\mathrm{THz}$ mode $A_{T H z}(z)$. The second term on the RHS of Eq. (1) corresponds to sum-frequency generation (SFG) between the $\mathrm{THz}$ and $(\mathrm{m}-1)^{\text {th }}$ optical mode. $\Lambda$ is the PPLN period, $\chi_{0}^{(2)}$ is the second-order susceptibility and $n\left(f_{m}\right)$ is refractive index at frequency $f_{m}$. The calculations consider hundreds of stokes and anti-stokes modes. Similarly, the evolution of the THz mode $A_{T H z}(z)$ is given by

$$
\frac{d A_{T H z}}{d z}=\frac{-\alpha}{2} A_{T H z}-\frac{j 2 f_{T H z} \chi_{0}^{(2)}}{c n\left(f_{T H z}\right)} \sum_{m=1}^{N-1} A_{m+1} A_{m}^{*} e^{-j\left(k_{m+1}-k_{m}-k_{T H z}+2 \pi \Lambda^{-1}\right) z}
$$

The first term on the RHS of Eq. (2) corresponds to $\mathrm{THz}$ absorption, while the second term corresponds to $\mathrm{THz}$ generation by the sum of all possible DFG processes between optical modes. We thus see how Eqs.(1)-(2) involve the coupled nonlinear interaction of multiple-triplets $\left(A_{m+1}, A_{m}, A_{T H z}\right)$ of waves. Only the first quasi-phase-matched order $\mathrm{THz}$ wave is considered. Equations (1)-(2) include dispersion in the optical region and $\mathrm{THz}$ absorption. For the relatively low intensities associated with the long pulse durations specific to this problem, self-phase modulation (SPM) effects may be omitted. In general, the conversion efficiency $(\eta)$ in the discrete case is the ratio of the total spectral intensities

$$
\eta(z)=\frac{n\left(f_{T H z}\right)\left|A_{T H z}\left(f_{T H z}, z\right)\right|^{2}}{\sum_{m} n\left(f_{m}\right)\left|A_{m}\left(f_{m}, 0\right)\right|^{2}}
$$
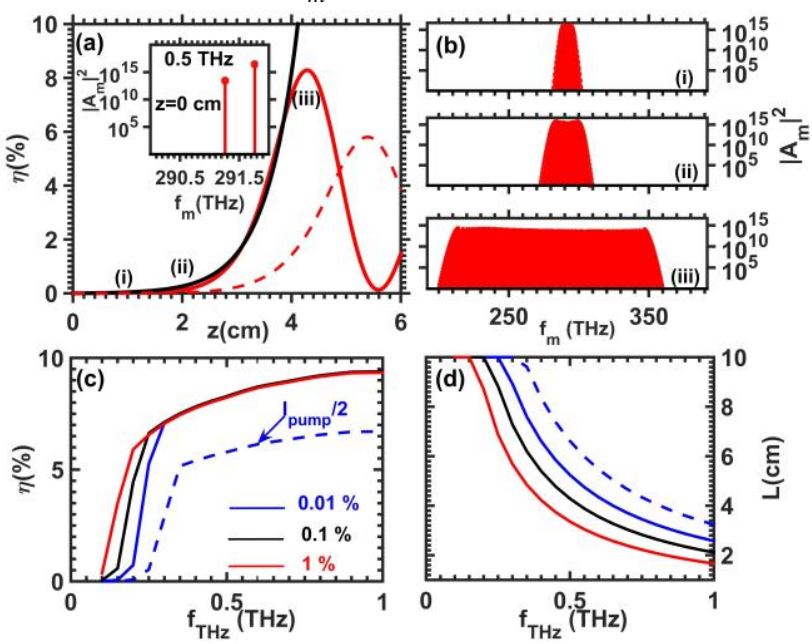

Fig.1 (a) Inset: Pump at $291.76 \mathrm{THz}$ with red-shifted seed with $0.1 \%$ of pump energy. Conversion efficiency $(\eta)$ versus crystal length (redsolid) is fit to hyperbolic sine growth (black). At half the pump intensity, $\eta$ grows slower with comparable maximum. A decline in $\eta$ due to alteration of phase-matching caused by spectral shift; (b) optical spectra at locations (i)-(iii) indicated in panel (a); (c) maximum $\eta$ as a function of seed level ( 0.01 to $1 \%$ of pump energy) and THz frequency at different pump intensities; (d) optimal crystal lengths corresponding to parameters panel (c).

The nonlinear material under consideration is magnesium oxide doped ( $5 \%$ mol.) congruent LN. The refractive indices and absorption coefficients are obtained from [21-23]. The optical modes span a frequency bandwidth from $f_{m}=100$ to $f_{m}=500 \mathrm{THz}$. Equations (1)-(3) are solved numerically via a $4^{\text {th }}$ order RungeKutta method with spatial resolution $\Delta z=0.5 \mu \mathrm{m}$. The bulk 
susceptibility $\chi_{0}^{(2)}=336 \mathrm{pm} / \mathrm{V}$. The maximum fluence, limited by laser-induced damage, is phenomenologically related to the $e^{2}$ pulse duration $\tau$ as $F_{d}=8.5(\tau / 10 \mathrm{~ns})^{1 / 2} \mathrm{Jcm}^{-2}$ [24]. This is expected to be similar at cryogenic temperatures due to insufficient alteration of the band gap to affect free-carrier generation. A PPLN crystal phase-matched for generation of 0.5 $\mathrm{THz}$ radiation with poling period $\Lambda=238 \mu \mathrm{m}$ at a cryogenic temperature of $77 \mathrm{~K}$ is assumed. As shown in the inset of Fig. 1a, the pump mode corresponding to a pulse with TL duration $\tau=100$ ps was located at $f_{0}=291.76 \mathrm{THz}$ (1028.2 nm, e.g. Yb: YAG lasers) with pump intensity $F_{d}(\tau \sqrt{\pi / 2})^{-1}$. The seed pulse, also corresponding to a TL duration $\tau=100 \mathrm{ps}$, is red-shifted by $0.5 \mathrm{THz}$ with respect to the pump with $0.1 \%$ of the pump intensity. For perfect spatial overlap, the seed will contain $0.1 \%$ of the pump energy, or $1 \mathrm{~mJ}$ for a $1 \mathrm{~J}$ pump.

In Fig. 1a, the conversion efficiency $\eta$ as a function of length for the aforementioned parameters is plotted (Fig. 1a, red-solid). For $z$ $<4 \mathrm{~cm}, \eta$ follows a sine hyperbolic ( exponential) fit (Fig. 1a, black). For $z>4 \mathrm{~cm}, \eta$ drops drastically before ascending again at $\sim 5.6 \mathrm{~cm}$. A second curve (Fig.1a, red-dashed) is plotted for half the maximum pump intensity. The behavior and peak efficiency is similar apart from a delayed onset of growth (or lower gain), similar to conventional OPAs. In Fig. 1b, the optical spectra at values of $z$ indicated by labels (i)-(iii) for the case of maximum pump intensity (Fig. 1a, red-solid) are shown. At (i) $z=1 \mathrm{~cm}$ and (ii) $z=2 \mathrm{~cm}$, before the onset of exponential growth, a modulation of the optical spectrum with roughly symmetric red (DFG) and blue-shift (SFG) is observed. During this initial stage, THz growth is not significant due to comparable rates of SFG and DFG. However, as THz radiation builds up significantly, a red-shift is preferred as evident at (iii) $z=4 \mathrm{~cm}$ and is accompanied by high conversion efficiencies in excess of $8 \%$. Thus, using THz-COPA, THz pulses with tens of mJs of pulse energy may be obtained with existing laser and crystal fabrication technology. The decline and subsequent oscillation of $\eta$ in Fig. 1a is attributed to the change in phase-matching conditions caused by the frequency shift and broadening of the optical spectrum. This may be remedied by rephasing the optical and $\mathrm{THz}$ radiation in a subsequent stage or varying the PPLN period along the crystal length.

In Fig. 1c, the maximum conversion efficiency achieved over PPLN crystal lengths $<10 \mathrm{~cm}$ for various $\mathrm{THz}$ frequencies and seed levels are depicted. The rate of energy growth for lower $\mathrm{THz}$ frequencies is lower, in line with expectations from conventional OPAs with a gain proportional to $\left(f_{f} f_{\mathrm{THz}}\right)^{1 / 2}$. Therefore, for a given seed level, frequencies beyond a threshold value are able to reach maximum conversion efficiency within the $10 \mathrm{~cm}$ length. Naturally, this threshold value decreases with increasing seed level or peak pump intensity. The corresponding optimal crystal lengths are plotted in Fig. 1d. Consistent with expectations, the optimal crystal lengths are shorter for larger THz frequencies, and higher seed levels and pump intensities (Fig. 1d, blue-dashed). For longer pulse durations $\tau$, the peak pump intensity reduces due to damage restrictions. In such cases, longer crystals or larger seed levels may be necessary.

In Fig. 2, we examine the evolution of optical spectra for various cases of phase-mismatch $\Delta k_{m}$ (Fig. 2a) to shed light on the physics of THz-COPA. Absorptive effects are switched off to not obfuscate the essential physics. In Fig. $2 \mathrm{~b}, \eta$ as a function of $z$ for cases (i)-(iii) of $\Delta k_{m}$ are shown. Case (i) maps the case of the PPLN simulated in Fig. 1, i.e., $\Delta k_{m}=k_{m+1}-k_{m}-k_{T H z}+2 \pi \Lambda^{-1}$. Here, we see that $\Delta k_{m}$ is almost symmetric for frequencies red and blue-shifted with respect to the pump frequency $f_{0}$ (Fig. 2a, blue). The corresponding $\eta$ versus $z$ curve (Fig. 2b, blue) resembles the red-solid curve in Fig. 1a. However, the values of $\eta$ are larger and an oscillation rather than decline is observed for $z>4 \mathrm{~cm}$ since absorption is neglected. In Fig. 2c, the optical spectrum as a function of $z$ is plotted for case (i). Here, an initial modulation of the optical spectrum, resulting in a large number of side-bands, blue and red-shifted with respect to $f_{0}$ is observed. Each SFG process consumes a THz photon, whereas each DFG process generates a THz photon. Consequently, during this initial stage, there is little growth in the efficiency. In contrast to conventional three-wave mixing OPAs, this behavior corresponds to a large ensemble of coupled three-wave $\left(A_{m+1}, A_{m}\right.$, $A_{\text {THz }}$ ) mixing processes. This is made possible by the fact, that $\mathrm{THz}$ frequencies are small in comparison to the phase-matching bandwidth of the material. Due to this competition between SFG and DFG, THz seeding in PPLN's may lead to initial THz attenuation before producing similar exponential growth.
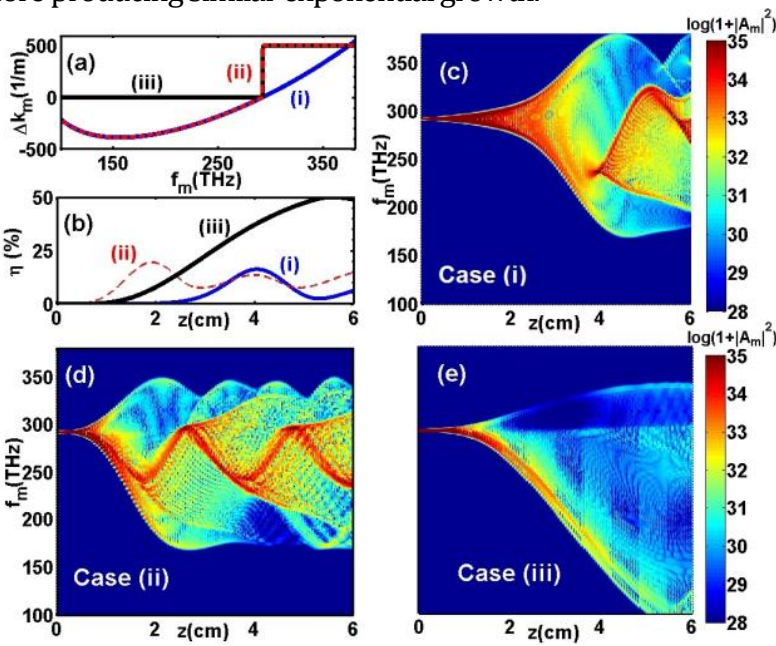

Fig.2 Role of phase-mismatch in THz-COPA: (a) Cases (i)-(iii) of phasemismatch $\Delta k_{m}$; (b) corresponding conversion efficiencies for $\Delta k_{m}$ cases (i)-(iii); (c) evolution of optical spectrum for case (i) showing initial modulation behavior; (d) evolution of optical spectrum for case (ii) showing predominant red-shift due to large phase-mismatch for blueshifted frequencies $\left(\Delta k_{m}=500 m^{-1}\right.$ for $\left.f_{m}>f_{0}\right)$; (e) evolution of the optical spectrum for case (iii) shows steady red-shift due to preservation of phase-matching conditions upon red-shift $\left(\Delta k_{m}=0\right.$ for $\left.f_{m}<f_{0}\right)$.

After initial symmetric broadening, the process shows a preferential red-shift due to marginally larger dispersion for frequencies larger than $f_{0}$ as seen in case (i) (Fig. 2a, blue). Correspondingly, the THz begins to grow exponentially (Fig. $2 \mathrm{~b}$, blue). We confirm our hypothesis by defining $\Delta k_{m}=500 \mathrm{~m}^{-1}$ for $f_{m}>f_{0}$, while leaving it unchanged for $f_{m}<f_{0}$ in case (ii) (Fig. 2a, reddashed). Correspondingly, the blue-shift in the spectrum is negligible (Fig. 2d), resulting in an earlier onset of exponential THz growth (Fig. 2b, red). However, for cases (i)-(ii), there is a saturation and oscillation of conversion efficiency beyond a certain length. This is due to the change in phase-matching conditions due to the frequency shift in the optical spectrum. To test this, we define $\Delta k_{m}=0$ for $f_{m}<f_{0}$ and retain $\Delta k_{m}=500 \mathrm{~m}^{-1}$ for $f_{m}>f_{0}$ in case (iii) (Fig. 2a, black). We then see that the conversion efficiency does not saturate but continues to grow over a longer portion of the crystal length (Fig. 2b, black). The optical spectrum for case (iii) exhibits a 
steady and predominant red-shift as delineated in Fig. 2e. The peak pump intensity for case (iii) is reduced to half the value of cases (i)(ii), so that maximum conversion efficiency is attained at $z=6 \mathrm{~cm}$. Therefore, case (iii) signifies that by varying the PPLN period along the propagation direction, such that $\Delta k_{m} \sim 0$ for $f_{m}<f_{0}, \eta>>10 \%$ could be achieved.

Figure 2 thus illustrates the need for discriminating between blue and red-shift. This behavior is markedly different from conventional OPAs, where large frequency differences between pump and signal, restrict the behavior to a three-wave mixing process. In the multi-triplet case, in the absence of any dispersion in the material, i.e., $\Delta k_{m}=0$, a mere oscillation of conversion efficiency as shown in Fig. 3a is observed. In Fig. $3 b$, the corresponding evolution of the optical spectrum is displayed. Symmetric broadening or modulation of the optical spectrum, with respect to the pump frequency $f_{0}$ is observed.
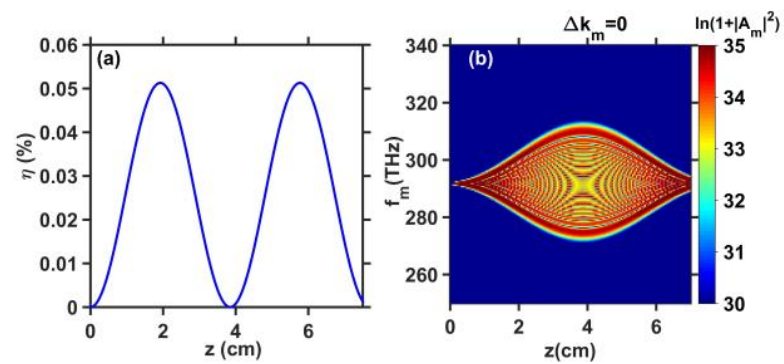

Fig. 3(a) For perfect phase-matching, i.e., $\Delta \mathrm{k}_{\mathrm{m}}=0$, conversion efficiency shows oscillatory behavior with respect to length. (b) Optical spectrum shows symmetric broadening due to modulation.

A simple analysis sheds light on this behavior: Suppose, only the pump $\left(A_{0}\right)$, two side-bands $f_{0} \pm f_{T H z}\left(A_{ \pm 1}\right)$ and the THz wave $\left(A_{T H z}\right)$ from Eqs. (1)-(2) are considered in the undepleted limit of the pump, oscillatory behavior is obtained for perfect phase matching.

$$
\begin{aligned}
& A_{T H z}(z)=A_{T H z, \max } \sin (\kappa z), \kappa=2 \sqrt{2} \chi_{0}^{(2)}\left|A_{0}\right| f_{T H z} c^{-1}\left(n\left(f_{0}\right) n\left(f_{T H z}\right)\right)^{-1 / 2} \\
& A_{ \pm 1}(z)=A_{ \pm}(0)+j 4\left(\operatorname{c\kappa }\left(f_{0}\right)\right)^{-1}\left(f_{0} \pm f_{T H z}\right) \chi_{0}^{(2)} A_{0} A_{T H z, \max } \sin ^{2}(\kappa z / 2)
\end{aligned}
$$

On the contrary, exponential growth is obtained in the three-wave case. Another example of interesting behavior is indifference to the location of the seed with respect to the pump. In conventional OPAs, the pump has the highest frequency compared to signal and idler. However, due to the multi-triplet nature of the THz-COPA, the initial spectrum rapidly washes out, thus also leading to exponential THz growth as shown in Fig. 4.

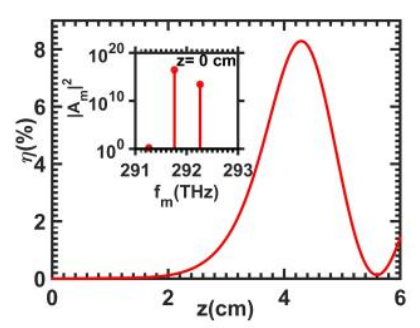

Fig.4. Indifference to seed location: Contrary to conventional OPAs, the THz-COPA shows exponential growth even if the seed is at higher frequency relative to the pump (inset).
In conclusion, a practical approach to high-energy THz generation based on spectrally cascaded optical parametric amplification (THz-COPA) was introduced. Starting with a strong narrowband optical pump and significantly weaker optical seed pulse, extensive frequency shifting due to cascading occurs which results in conversion efficiencies at the $\sim 10 \%$ level in cryogenically cooled PPLN. Conversion efficiencies $>>10 \%$ may be obtained by multistaging or varying the PPLN period along the crystal. The THzCOPA exhibits distinctively different physics from conventional OPAs. This influences the optimal pulse formats and phasematching conditions. The approach paves the way for extremely high energy THz sources.

Funding European Research Council (grant n. 609920); "The Hamburg Centre for Ultrafast Imaging- Structure, Dynamics and Control of Matter at the Atomic Scale" of the Deutsche Forschungsgemeinschaft (grant EXC 1074).

\section{References}

1. L. J. Wong, A. Fallahi, and F. X. Kärtner, Opt. Express 21,9792 (2013).

2. E.A. Nanni, W.R. Huang, K.H. Hong, K. Ravi, A. Fallahi, G. Moriena, R.J.D. Miller, and F.X. Kärtner, Nat. Comm. 6:8486 (2015).

3. F.X.Kärtner, Nucl. Inst. and Meth. in Phys. Res. A 829,24(2016).

4. N. Yardimici, S.-H. Yang, C. W. Berry and M. Jarrahi, IEEE Trans. THz Sci. Tech. 5, 223 (2015).

5. S. Gold, and G. Nusinovich, Rev. Sci. Instr. 68, 3945 (1997).

6. G. P. Gallerano, and S. Biedron, Proc. FEL conf., 216 (2004).

7. K. L. Vodopyanov, Laser \& Photonics Rev. 2, 11 (2008).

8. K. L. Vodopyanov, Appl. Phy. Lett. 89, 141119 (2006).

9. G. Kitaeva, Laser Phys. Lett. 5, 559 (2008).

10. J. Lu, H. Hwang, X. Li, S.-H. Lee, O. Pil-Kwon, and K. A. Nelson, Opt. Express, 23, 22723 (2015).

11. Z. Chen, X. Zhou, C. Werley, and K. A. Nelson, Appl. Phy. Lett. 99, 071102 (2011).

12. K. Ravi, W. Huang, S. Carbajo, X. Wu, and F. X. Kärtner, Opt. Express, 22, 20239 (2014).

13. S. Carbajo, J. Schulte, W. Xiaojun, K. Ravi, D. Schimpf, and F. X. Kärtner, Opt. Lett. 40, 5762 (2015).

14. M. C. Golomb, Opt. Lett. 29, 2046 (2004).

15. L. Zapata, et al, Opt. Lett. 40, 2610 (2015).

16. T. Taira and, H. Ishizuki, Opt. Express 22, 19668 (2014).

17. S. R. Tripathi, Y. Taira, S. Hayashi, K. Nawata, K. Murate, H. Minamide and K. Kawase, 39, 1649 (2014).

18. D. Molter, M. Theuer and R. Beigang, Opt. Express 17, 6623 (2009).

19. D. Walsh, P. Browne, M. Hunn, and C. Rae, Opt. Express 18, 13951 (2010).

20. I.Jovanovic, C.P.J.Barty, C.Haefner, and B.Wattellier, Opt.Lett. 31,787 (2006)

21. D. H. Jundt, Opt. Lett. 22, 1553 (1997).

22. L. Palfalvi, J. Hebling, J. Kuhl, A. Peter and K. Polgar, J. Appl. Phys. 97, 123505 (2005).

23. J. A. Fülöp, L. Palfalvi, M. Hoffmann and J. Hebling, Opt. Express 19, 15090 (2011).

24. V. G. Dmitriev, G. G. Gurzadyan, and D. N. Nikogosyan , Handbook of Nonlinear Crystals (Springer Verlag, 1999). 